Article

\title{
On the Applicability of the Green Chemistry Principles to Sustainability of Organic Matter on Asteroids
}

\author{
Vera M. Kolb \\ Department of Chemistry, University of Wisconsin-Parkside, Kenosha, WI 53141-2000, USA; \\ E-Mail: kolb@uwp.edu; Tel.: +1-262-595-2133; Fax: +1-262-595-2056.
}

Received: 20 April 2010; in revised form: 27 May 2010 / Accepted: 2 June 2010 /

Published: 4 June 2010

\begin{abstract}
The connection between astrobiology and green chemistry represents a new approach to sustainability of organic matter on asteroids or similar bodies. Green chemistry is chemistry which is environmentally friendly. One obvious way for chemistry to be green is to use water as a solvent, instead of more toxic organic solvents. Many astrobiological reactions occur in the aqueous medium, for example in the prebiotic soup or during the aqueous alteration period on asteroids. Thus any advances in the green organic reactions in water are directly applicable to astrobiology. Another green chemistry approach is to abolish use of toxic solvents. This can be accomplished by carrying out the reactions without a solvent in the solventless or solid-state reactions. The advances in these green reactions are directly applicable to the chemistry on asteroids during the periods when water was not available. Many reactions on asteroids may have been done in the solid mixtures. These reactions may be responsible for a myriad of organic compounds that have been isolated from the meteorites.
\end{abstract}

Keywords: green chemistry; astrobiology; solventless reactions; solid-state reactions; Diels-Alder reactions in water; asteroids; meteorites

\section{Introduction}

The goals of sustainability and astrobiology are intertwined, as noted in the NASA's Astrobiology Roadmap, for example [1]. Especially relevant is the Roadmap's Goal 2, to determine past prebiotic chemistry and the history of chemical ingredients, which are important for habitable environments and which could sustain living systems. Myriads of organic compounds have been found on meteorites 
which are carbonaceous chondrites, such as Murchison [2-10]. Tens of thousands of different molecular compositions were revealed on Murchison by the application of ultra-high resolution analytical methods which combine state-of-the-art mass spectrometry, liquid chromatography and nuclear magnetic resonance. Meteorites are obtained from meteors, and the latter from asteroids [2-4]. Chemistry on asteroids, as implied by the organic chemicals that were identified on meteorites, is not fully understood, but is considered important for the early chemical evolution [11-14]. As opposed to the situation on the early Earth, where water was consistently available, water on asteroids was available only during the periods of so-called aqueous alteration [2]. While it is acknowledged that chemicals may be deposited on asteroids or other extraterrestrial bodies via comets, interstellar dust particles, collisions with other bodies, and similar means, it is not clear how these chemicals would progress along the lines of chemical evolution without a constant availability of water. To aggravate the situation further, most organic materials are not water soluble. Thus, even though water became periodically available, it is not clear how this would help the water-insoluble materials to react.

Some of the problems of the organic synthesis on asteroids or similar bodies can be solved by borrowing the knowledge from a new and emerging field of green chemistry [15-18]. The tools and principles of green chemistry are remarkably applicable to these astrobiological problems. They could complement and enrich the already proposed synthetic ways. For example, the reactions on asteroids could have occurred by adsorption and subsequent catalysis on the solid surface of zeolites, clays, silica, alumina and similar substances. The green chemistry solventless and solid-solid reactions provide an additional alternative. On the other hand, the reactions on asteroids which may not be thermally activated, but instead are activated by the circularly polarized light, or radioactive decay, at this stage would not benefit from the green chemistry.

In this paper we focus on two areas of green chemistry, the reactions of organic materials in water via hydrophobic interactions and the solventless and solid state organic reactions.

\section{Aqueous Reactions of Water-Insoluble Organic Materials}

Aqueous reactions that we consider in this sections and are not those of the organic compounds that are water soluble; instead, they involve water-insoluble organic components that are highly hydrophobic. One such reaction is Diels-Alder reaction [19]. This reaction occurs between a diene and a dienophile. It is a major reaction that forms carbon-carbon bond. It produces six-membered rings and various relatively complicated molecules in a single step, and is very efficient [19]. We show one example below. The dashed lines show the required orbital overlap, which leads to the stereospecificity of the reaction (the endo isomer, which is shown, is the preferred isomer). 
Figure 1. An Example of Diels-Alder Reaction.
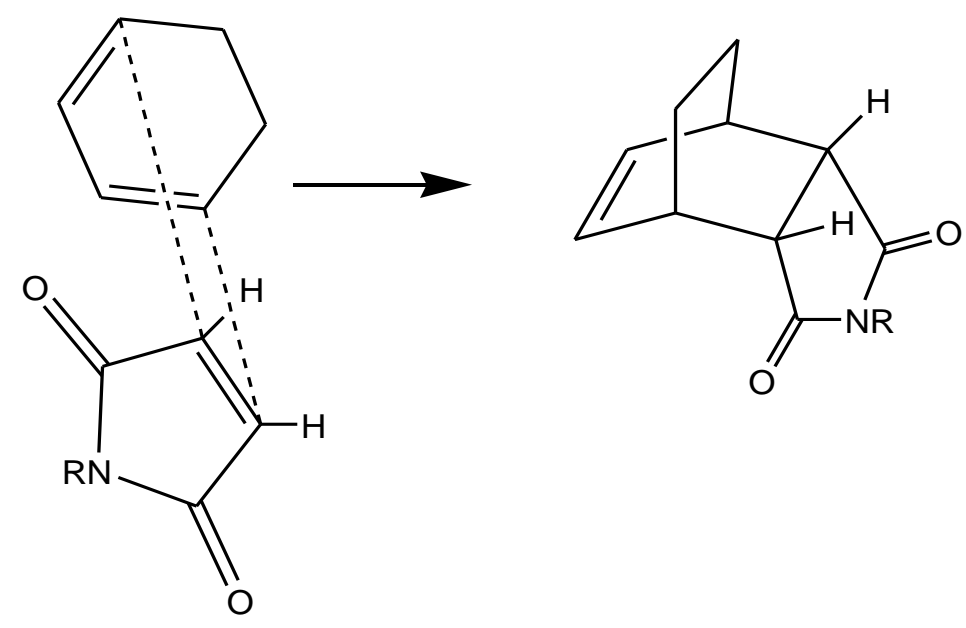

The Diels-Alder reaction is traditionally performed in an organic solvent, but was found to occur also in the aqueous solution [20-22]. The way the reaction occurs in water is the following. The water-insoluble (hydrophobic) reactants, when placed in water, are driven towards each other so that they avoid water. This is a so-called hydrophobic interaction, which is well known in biology, notably for the interaction of peptides [23]. When two organic components, a diene and a dienophile, are driven towards each other, the chance of a proper alignment of their orbitals, which is necessary for the reaction to occur, increases. When the reaction is performed, as normally is, in an organic solvent, the reactants dissolve in the solvent and are thus diluted. This diminishes their chance for the encounter, and, consequently, the reaction rate. It also affects adversely the endo-specificity of the reaction. Thus, the Diels-Alder reaction in water is not only feasible, but is also faster and more specific [20-22]. The organic solvents were not present as such on the prebiotic Earth or asteroids. However, water was present on the former and also periodically on the latter, during the period of aqueous alteration [2]. Thus, the Diels-Alder reaction in water is prebiotically feasible. The hydrophobic interaction as a driving force for the reaction of water-insoluble organic materials can be extended in principle to other organic reactions. When put into the experimental practice, it may provide a way for other such reactions to become prebiotically feasible. Diels-Alder reaction has not been fully explored for its prebiotic synthetic potential. However, this reaction has been studied as a model for prebiotic molecular self-replication first by Wang and Sutherland [24]. Their study was followed up by others (e.g., [25]). Wang and Sutherland devised a self-replicating system based on the diene and dienophile components, which were not particularly reactive until they were attached to the chemical moieties, designed to have specific hydrogen-bonding patterns. Such attachments led to an increase in the reaction rate and to the sigmoidal character of the kinetic curve, which signified the autocatalytic reaction. The structures which depict and summarize the Wang-Sutherland model are shown below. The Diels-Alder reaction product is shown on the top, while the diene and dienophile are shown underneath. The diene and dienophile have attachments that allow precise hydrogen bonding which becomes complementary with that in the product. The product thus acts as a molecular template to bring together and properly align the diene and dienophile via the hydrogen bonding between the attachments. The hydrogen bonding is shown as dashed lines. The product catalyzes the formation of more products. 
Figure 2. An Example of Diels-Alder Self-replicator.

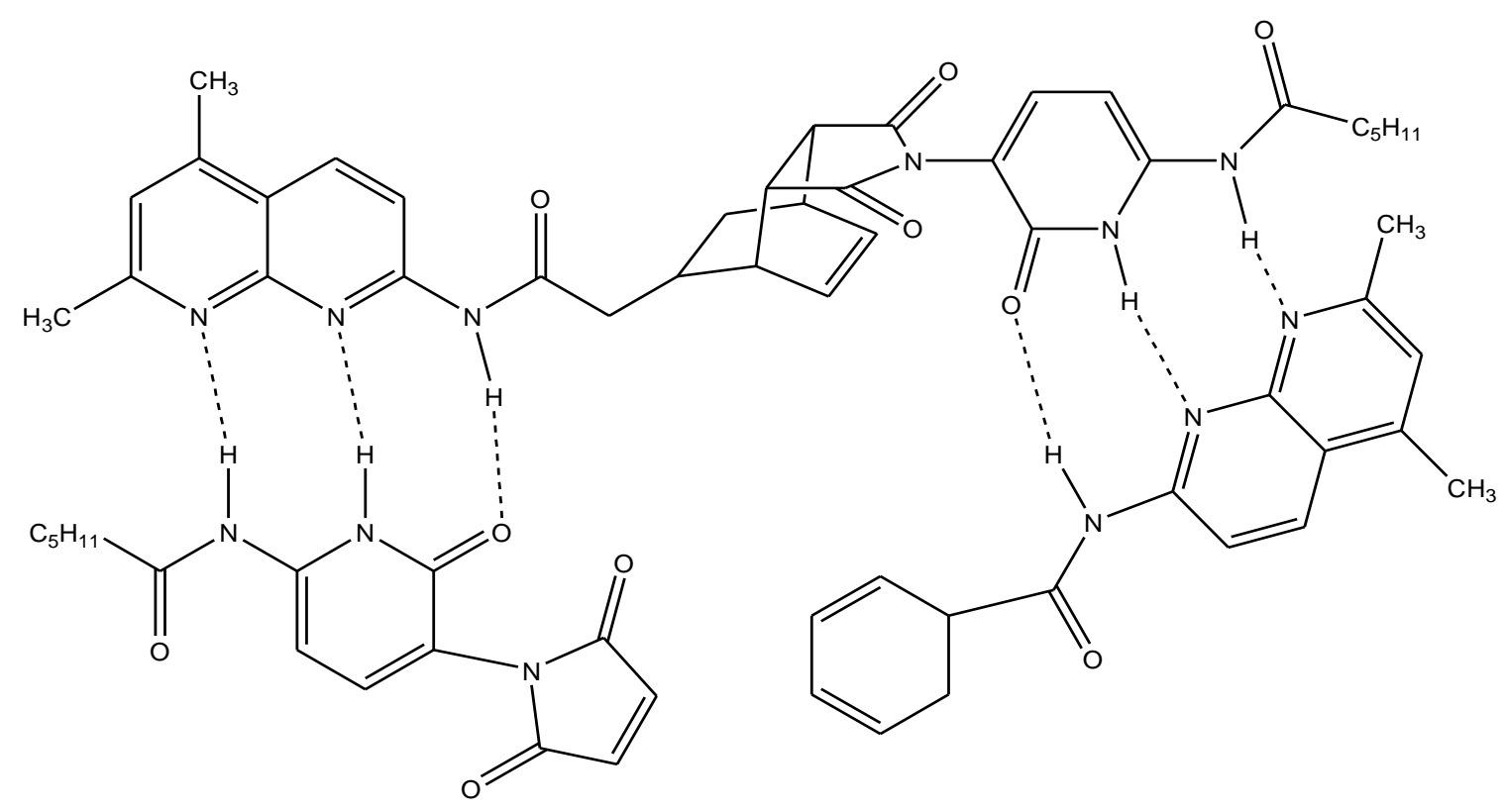

This self-replicating model system works only with the specific attachments, which are not likely to be spontaneously synthesized under the prebiotic conditions. It utilizes a non-prebiotic solvent (deuterated chloroform or methylene chloride), which was necessitated by the use of the nuclear magnetic resonance to study the kinetics of the reaction. However, this model may still be useful in specific prebiotic niches. If we would place the above model system in water, thus green solvent, the hydrogen bonding pattern which are necessary for the alignment of the diene and dienophile on the template would be disrupted by water. However, in water the diene and dienophile, without any attachments, could align themselves properly without any template because of the hydrophobic effect. The use of water as solvent would detract from the self-replication aspect of this Diels-Alder model, but it would enhance its synthetic aspect in this medium.

\section{Solventless and Solid-State Organic Reactions}

The second green chemistry area that is relevant to the chemistry on asteroids is that of the solventless and solid-state reactions. A substantial body of the literature illustrates that many organic reactions occur in the solid state [26-32], often rapidly and with remarkable efficiency [27,28]. Examples include all major types of organic reactions, such as oxidations, reductions, eliminations, substitutions, condensations, cyclizations, rearrangements, and many others. Detailed experimental procedures for solvent-free reactions are compiled also in a recent book [32]. The principles of the solid state reactions imply feasibility of the solid-state reactions on asteroids. The organic materials that would be formed in the solid-state reactions on asteroids would have a chance to react further during the periods of aqueous alterations [2]. This double process could potentially account for the great richness in the variety of the organic chemicals that are found on meteorites, comprising of well over 400 types of compounds by the earlier findings [2] to tens of thousands by the most recent measurements [5]. 
We describe here a remarkably simple principle behind some of the solid state reactions, the so-called solventless reactions [30,33]. The principle is that of the lowering of the melting point of a pure component, when another component is added to it [30,33]. When the solid organic compound is impure, its melting point is lowered. This is due to the incorporation of the impurities into the crystalline lattice of the compound, which breaks up the regular crystalline pattern. When more impurities are present, the melting point becomes even lower. The ingenious design of the solid-state reactions which exploits this principle is the following. One takes two solid reactants and mixes or grinds them together. This causes the lowering of the melting point. If the reactants are chosen well, the melting point will be lowered to the room temperature, thus giving a viscous liquid. The reaction will then occur in this liquid phase [30,32]. This process eliminates the need for the solvent. Because of this, such solid state reactions are known as "solventless" [30]. This could be a possible scenario on asteroids, where there were many compounds, actually a myriad judging by the analysis of Murchison and similar carbonaceous chondrites [2-10]. Some of these compounds may be reactive towards each other, but others may not be. However, the unreactive compounds could lower the melting points of the reactive ones, enabling the reactions to occur in the melted state. The temperature estimates on the asteroids include a range from $25{ }^{\circ} \mathrm{C}$ to $100{ }^{\circ} \mathrm{C}$, which is consistent with aqueous alterations [2] and is friendly to the organic chemicals reactions and preservation of the organic compounds. The heat is provided by the radioactive processes or impacts [2].

There are more than 30 types of major organic reactions [28-32] that have been found to occur in the solid state with supreme efficiency and the yields of $100 \%$ [28]. Not all of these solid state reactions occur by the mixed melting point liquefaction principle. Some occur by simply mixing the solid reactants and adding a solid or gaseous catalyst, without ever melting the reactions mixture. Especially interesting is the formation of ethers in the solid state, from the solid mixture of the alcohols and acid catalysts [31]. Such a synthesis could be a prebiotically feasible way to produce various ethers, which primitive organisms could just absorb from the environment. This could be relevant to the use of the ether-based lipids in some archaea's membranes (as opposed to the use of ester-type lipids by other organisms) [33-35].

\section{Conclusions}

In this paper we show how selected green chemistry principles and tools can be useful in accounting for chemical synthesis on asteroids, specifically between the organic compounds that are not water soluble. We address the solid-state and solventless reactions, as well as the reactions in the aqueous medium which are driven by the hydrophobic effect, such as the Diels-Alder reaction. These reactions can explain in principle the sustainability of organic materials on asteroids.

\section{Acknowledgements}

Thanks are expressed to Wisconsin Space Grant Consortium/NASA for sponsoring our efforts of infusion of astrobiology into the organic chemistry curriculum, which have inspired this paper. Comments by the reviewers are gratefully acknowledged. 


\section{References}

1. Des Marais, D.J.; Nuth, J.A., III; Alamandola, L.J.; Boss, A.P.; Farmer, J.D.; Hoehler, T.M.; Jakosky, B.M.; Meadows, V.C.; Pohorille, A.; Runnegar, B.; Spormann, A.M. The NASA astrobiology roadmap. Astrobiology 2008, 8, 715-730.

2. Kerridge, J.F. Formation and processing of organics in the early solar system. Space Sci. Rev. 1999, 90, 275-288.

3. Shaw, A.M. Meteorite and Comet Chemistry. In Astrochemistry: From Astronomy to Astrobiology; Wiley: New York, NY, USA, 2006; Chapter 6, pp. 157-192.

4. Cronin, J.R. Clues from the Origin of the Solar System: Meteorites. In The Molecular Origins of Life; Brack, A., Ed.; Cambridge University Press: Cambridge, UK, 1998; pp. 119-146.

5. Schmitt-Kopplin, P.; Gabelica, Z.; Gougeon, R.D.; Fekete, A.; Kanawati, B.; Harir, M.; Gebefuegi, I.; Eckel, G.; Hertkorn, N. High molecular diversity of extraterrestrial organic matter in Murchison meteorite revealed 40 years after its fall. Proc. Nat. Acad. Sci. USA 2010, 107, 2763-2768.

6. Sephton, M.A. Organic compounds in carbonaceous meteorites. Nat. Prod. Rep. 2002, 19, 292-311.

7. Sephton M.; Gilmour, I. Macromolecular Organic Materials in Carbonaceous Chondrites: A Review of Their Sources and Their Role in the Origin of Life on the Early Earth. In Impacts and the Early Earth; Gilmour, I., Keoberl, C., Eds.; Springer-Verlag: Berlin, Germany, 2000; pp. 27-49.

8. Cronin, J. R.; Chang, S. Organic Matter in Meteorites: Molecular and Isotopic Analysis of the Murchison Meteorite. In The Chemistry of Life's Origins; Greenberg, J.M., Mendoza-Gomez, C.X., Pirronello, V., Eds.; Kluwer Academic Publishers: Dodrecht, The Netherlands, 1993; pp. 209-258.

9. Cronin, J.R.; Cooper, G.W.; Pizzarello, S. Characteristics and formation of amino acids and hydroxyl acids of the Murchison meteorite. Adv. Space Res. 1995, 15, 91-97.

10. Cooper, G.; Kimmich, N.; Belisle, W.; Sarinana, J.; Brabham, K.; Garrel, L. Carbonaceous meteorites as a source of sugar-related organic compounds for the early Earth. Nature 2001, 414, 879-883.

11. Shaw, A.M. Prebiotic Chemistry. In Astrochemistry: From Astronomy to Astrobiology; Wiley: New York, NY, USA, 2006; Chapter 8, pp. 225-255.

12. Lurquin, P.F. The Origins of Life and the Universe; Columbia University Press: New York, NY, USA, 2003.

13. Fry, I. The Emergence of Life on Earth; Rutgers University Press: New Brunswick, NJ, USA, 2000.

14. Luisi, P.L. The Emergence of Life; Cambridge University Press: Cambridge, UK, 2006.

15. Doxsee, K.M.; Hutchinson, J.E. Green Organic Chemistry, Strategies, Tools, and Laboratory Experiments; Thomson Brooks/Cole: Toronto, Canada, 2004.

16. Anastas, P.T.; Warner, J.C. Green Chemistry: Theory and Practice; Oxford University Press: Oxford, UK, 2000. 
17. Lancaster, M. Green Chemistry, an Introductory Text; Royal Society of Chemistry: Cambridge, UK, 2002.

18. Lankey, R.L.; Anastas, P.T. Advancing Sustainability through Green Chemistry and Engineering; ACS Symposium Series 823; American Chemical Society: Washington, DC, USA, 2002.

19. Laue, T.; Plagens, A. Named Organic Reactions, 2nd ed.; Wiley: Chichester, UK, 2005; pp. 88-95.

20. Breslow, R. Hydrophobic effects on simple organic reactions in water. Acc. Chem. Res. 1991, 24, 159-164.

21. Blokzijl, W.; Blandamer, M.J.; Engberts, J.B.F.N. Diels-Alder reactions in aqueous solutions. Enforced hydrophobic interactions between Diene and Dienophile. J. Am. Chem. Soc. 1991, 113, 4241-4246.

22. Meijer, A.; Orro, S.; Engberts, J.B.F.N. Effect of the hydrophobicity of the reactants on Diels-Alder reactions in water. J. Org. Chem. 1998, 63, 8989-8994.

23. Voet, D.; Voet, J.G.; Pratt, C.W. Fundamentals of Biochemistry: Life at the Molecular Level; Wiley: Hoboken, NJ, USA, 2006; pp. 27-28.

24. Wang, B.; Sutherland, I.O. Self-replication in a Diels-Alder reaction. Chem. Commun. 1997, 1495-1496, DOI:10.1039/a701573i.

25. Kindermann, M.; Stahl, I.; Reinhold, M.; Pankau, W.M.; von Kiedrowski, G. Systems Chemistry: Kinetic and Computational Analysis of a Nearly Exponential Organic Replicator. Angew. Chem. Int. Ed. Engl. 2005, 44, 6750-6755.

26. Tanaka, K.; Toda, F. Solvent-free organic syntheses. Chem. Rev. 2000, 100, 1025-1074.

27. Toda, F. Solid state organic chemistry: Efficient reactions, remarkable yields, and stereoselectivity. Acc. Chem. Res. 1995, 28, 480-486.

28. Kaupp, G. Organic Solid-state Reactions with $100 \%$ Yield. In Topics in Current Chemistry; Springer Berlin/Heidelberg: Heidelberg, Germany, 2005; Volume 254, pp. 95-183.

29. Cave, G.W.V.; Raston, C.L.; Scott, J.L. Recent advances in solventless organic reactions: Towards benign synthesis with remarkable versatility. Chem. Commun. 2001, 2159-2169, DOI:10.1039/b106677n.

30. Rothenberg, G.; Downie, A.P.; Raston, C.L.; Scott, J.L. Understanding solid/solid organic reactions. J. Am. Chem. Soc. 2001, 123, 8701-8708.

31. Toda, F.; Takumi, H.; Akehi, M. Efficient solid-state reactions of alcohols: Dehydration, rearrangement, and substitution. J. Chem. Soc. Chem. Commun. 1990, 1270-1271, DOI:10.1039/C39900001270.

32. Tanaka, K. Solvent-Free Organic Synthesis, 2nd ed.; Wiley-VCH: Weinheim, Germany, 2009.

33. Doxsee, K.M.; Hutchinson, J.E. Green Organic Chemistry, Strategies, Tools, and Laboratory Experiments; Thomson, Brooks/Cole: Toronto, Canada, 2004; pp. 115-119.

34. De Rosa, M.; Gambacorta, A.; Gliozzi, A. Structure, Biosynthesis, and Physicochemical Properties of Archaebacterial Lipids. Microbiol. Rev. 1986, 50, 70-80. 
35. Kates, M. Membrane Lipids of Archaea. In The Biochemistry of Archaea (Archaebacteria); Kates, M., Kushner, D.J., Matheson, A.T., Eds.; Elsevier: Amsterdam, The Netherlands, 1993; pp. 261-295.

(C) 2010 by the authors; licensee MDPI, Basel, Switzerland. This article is an Open Access article distributed under the terms and conditions of the Creative Commons Attribution license (http://creativecommons.org/licenses/by/3.0/). 